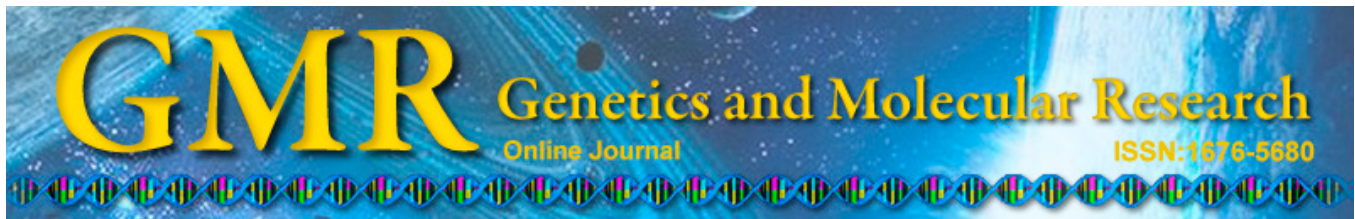

\title{
Protection effect of atorvastatin in cerebral ischemia-reperfusion injury rats by blocking the mitochondrial permeability transition pore
}

\author{
T. Song, J. Liu, X. Tao and J.G. Deng \\ Department of Neurological Rehabilitation, \\ Mawangdui Hospital of Hunan Province, \\ Hunan Provincial Research Institute of Geriatrics, Changsha, China \\ Corresponding author: X. Tao \\ E-mail: xitaocn@163.com
}

Genet. Mol. Res. 13 (4): 10632-10642 (2014)

Received July 25, 2014

Accepted November 5, 2014

Published December 18, 2014

DOI http://dx.doi.org/10.4238/2014.December.18.5

\begin{abstract}
The aim of this study was to investigate the influence of atorvastatin on the opening of the mitochondrial permeability transition pore (MPTP) and the expression of cytochrome C (Cyt C) in SpragueDawley rats with cerebral ischemia-reperfusion (I/R). The rat model of cerebral artery ischemia was established by the suture-occluded method with ischemia for $2 \mathrm{~h}$ and reperfusion for $72 \mathrm{~h}$. Thirty-four male rats were randomly divided into four groups: the normal group and the sham-operation group without any treatment, the I/R group with only intragastric administration of normal saline, and the intervention group, which received intragastric administration of $10 \mathrm{mg} / \mathrm{kg}$ atorvastatin at different times. All rats were sacrificed at $72 \mathrm{~h}$. Compared with the $\mathrm{I} / \mathrm{R}$ group, the morphology of nerve cells in the intervention group was reduced, the number of TUNEL-positive cells decreased, the expression of cortical cytoplasm $\mathrm{Cyt} \mathrm{C}$ decreased, and the mitochondrial absorbance value increased. All of these differences were statistically significant. Atorvastatin could inhibit neuronal apoptosis and alleviate the cerebral I/R injury. The mechanism may be related to the blocking
\end{abstract}


of the MPTP opening and the subsequent reduction of Cyt $\mathrm{C}$ release.

Key words: Atorvastatin; Mitochondrial permeability transition pore; Cytochrome C; Cerebral ischemia and reperfusion; Apoptosis

\section{INTRODUCTION}

Statins are the inhibitors of 3-hydroxy-3-glutaryl coenzyme A (HMG-CoA) reductase, which not only play a large role in lowering cholesterol, but also have multi-directional functions such as cholesterol-independent anti-inflammatory, cell proliferation and differentiation, anti-excitement toxins, anti-oxidation, anti-platelet aggregation, neuroprotective, and immunomodulatory activities, and are therefore one of the key drug types used to reduce the occurrence of clinical cardiovascular events (Bösel et al., 2005; Nahrendorf et al., 2008; Pignatelli et al., 2012; Zhang et al., 2012). Statins have a wide range of positive effects for ischemic diseases, and an increasing number of in-depth studies have focused on their protective mechanism. Owing to differences in the efficacy and side effects of different statin drugs, some of the drugs have gradually been clinically eliminated. As a second-generation HMG$\mathrm{CoA}$ reductase inhibitor, atorvastatin is currently widely used in the treatment of ischemic cerebrovascular diseases or for the primary prevention and secondary prevention of transient ischemia, and its application has shown positive results. However, the mechanism of action of statins is still not completely understood.

The mitochondrion is the major organ of energy metabolism, providing ATP for various cellular activities through oxidative phosphorylation. Mitochondria cannot effectively carry out oxidative phosphorylation in ischemic and hypoxic tissues, and thus $\mathrm{H}^{+}$accumulates, calcium overloads, the mitochondrial membrane potential becomes reduced, the permeability transition pore (MPTP) opens, and the mitochondria swell, which ultimately triggers apoptosis of the mitochondrial pathway. Recent studies have found that mitochondria can be important neuroprotective targets for cerebral ischemia-reperfusion (I/R) injury (Ye et al., 2012; Zhao et al., 2013). However, in the field of cerebral ischemia and reperfusion, there are few reports investigating whether the neuroprotective effects of ischemic stress by atorvastatin are associated with mitochondrial morphology and function. Therefore, in this study, the rat middle artery cerebral occlusion (MACO) model was established by the suture-occluded method, 10 $\mathrm{mg} / \mathrm{kg}$ atorvastatin was intragastrically administered, and the changes in MPTP opening and cytosolic cytochrome $\mathrm{C}$ (Cyt C) expression were observed in different experimental groups. These results were combined with observations of hematoxylin and eosin (HE) and TUNEL staining of brain tissues in order to elucidate the neuroprotection mechanism of atorvastatin.

\section{MATERIAL AND METHODS}

\section{Grouping and intervention of experimental animals}

Thirty-four pathogen-free male Sprague-Dawley (SD) rats weighing 220-250 g were provided from the Hunan Slack King of Laboratory Animal Limited Company (certificate No. SCXK (Xiang) 2009-0004). The 34 male rats were numbered and randomly divided according to the random number table into the normal control group (7 rats), the sham-operation group (7 rats), the I/R group (10 rats), and the atorvastatin intervention group (10 rats). In the sham- 
operation group, the neck skin of the rat was cut and the vessels were separated, but there was no drug treatment administered. In the I/R group, the rat MACO model was prepared, normal saline was intragastrically administered at the time of waking after reperfusion, and then at $24 \mathrm{~h}$ and again at $48 \mathrm{~h}$. In the atorvastatin intervention group, $10 \mathrm{mg} / \mathrm{kg}$ atorvastatin (Pfizer Company, USA), prepared with normal saline, was intragastrically administered at the same time points as that of the $\mathrm{I} / \mathrm{R}$ group. This study was carried out in strict accordance with the recommendations in the Guide for the Care and Use of Laboratory Animals of the National Institutes of Health. The animal use protocol was reviewed and approved by the Institutional Animal Care and Use Committee (IACUC) of the Mawangdui Hospital of Hunan Province.

\section{MACO model preparation}

The MACO model was prepared by the suture-occluded method (Clark et al., 1997). After the rats were weighed, they were intraperitoneally injected with $350 \mathrm{mg} / \mathrm{kg} 10 \%$ chloral hydrate anesthetic. Rats were then placed in supine position and their limbs were fixed, the soft tissues in the middle right side of the neck were separated to expose the right general carotid artery and the carotid artery, the proximal heart end of the general carotid artery and the root of the outward carotid artery were ligated, the internal carotid artery was clipped using arterial clamps, sutures were prepared, and a notch was cut near the bifurcation of the carotid artery. The special sutures (Beijing Sunbio Biotech Co., Ltd., Beijing, China) were inserted, and the artery clips were loosened; the insert depth was approximately $18-20 \mathrm{~mm}$ and was stopped when slight resistance was felt. The spare lines were tied, the skin was sutured, and then the suture was pulled out after ischemia for $2 \mathrm{~h}$ and reperfusion for $72 \mathrm{~h}$. Animals that died during surgery due to subarachnoid hemorrhage or neurological function impairment, which had unqualified scores, were considered as failed model preparations, and were not included in the analysis. The model number of any dead rat was complemented after model preparation of the same batch of rats.

\section{Neurological function impairment scoring}

Rats were subjected to neurological function scoring after waking from anesthesia according to the Longa 5-point scale standard (Longa et al., 1989) as follows: no symptoms of nerve injury ( 0 points), could not fully extend the contralateral paw (1 point), contralateral circling ( 2 points), contralateral dumping ( 3 points), could not spontaneously walk or loss of consciousness (4 points). Rats with scores of 0 or 4 points were excluded from the analysis.

\section{HE staining}

At 72-h reperfusion, the rats were intraperitoneally anesthetized with $350 \mathrm{mg} / \mathrm{kg} 10 \%$ chloral hydrate, and all rats were killed. Two rats from the normal group and the sham group, and five rats from the other groups were selected, respectively, and were cardiac perfused with $250 \mathrm{~mL} \mathrm{4 \%}$ paraformaldehyde phosphate-buffered saline (PBS) until the liver turned white. The brain tissues were then soaked in $4 \%$ paraformaldehyde PBS buffer at $4^{\circ} \mathrm{C}$ overnight, which were then subjected to graded alcohol dehydration, cleared with xylene, embedded in paraffin, and sliced into 5- $\mu \mathrm{m}$ sections for subsequent use.

Changes in nerve cell morphology were observed by HE staining. Sections were 
baked at $60^{\circ} \mathrm{C}$ for $30 \mathrm{~min}$ in the oven, dewaxed with xylene I for $5 \mathrm{~min}$, cleared with xylene II for $10 \mathrm{~min}$, and treated by graded alcohol for 2 to $4 \mathrm{~min}(100 \rightarrow 95 \rightarrow 85 \rightarrow 75 \%)$, respectively. The alcohol was washed away with distilled water, and immersed in hematoxylin for $5 \mathrm{~min}$, rinsed with distilled water for $2 \mathrm{~min}$, and the color was separated by using $1 \%$ hydrochloric acid alcohol for 10-20 s (several lifts and thrusts until the slices faded to pale blue), soaked in water for $10 \mathrm{~min}$, stained with eosin for $5 \mathrm{~min}$, dipped in distilled water a few times, and then re-dehydrated by graded ethanol $(75 \rightarrow 85 \rightarrow 95 \rightarrow 100 \%)$ for $5 \mathrm{~min}$, respectively, dewaxed in xylene I for $3 \mathrm{~min}$, cleared with xylene II for $5 \mathrm{~min}$, sealed in neutral gum, dried in a $37^{\circ} \mathrm{C}$ oven, and finally, the pathological changes of brain tissues were observed under the microscope. Eight different fields of view of each slice were randomly observed under high magnification (400X).

\section{TUNEL staining}

The TUNEL-Staining Kit was purchased from the Beyotime Institute of Biotechnology (Jiangsu, China). The following operation was carried out according to the kit instructions: the paraffin slices were dewaxed in xylene for $10 \mathrm{~min}$, and then further dewaxed in fresh xylene for 5-10 min, washed in the alcohol gradient (70-100\%) for $2 \mathrm{~min}$, respectively, and $20 \mu \mathrm{g} / \mathrm{mL}$ free DNase enzyme proteinase $\mathrm{K}$ was added, incubated at $37^{\circ} \mathrm{C}$ for $15-30 \mathrm{~min}$, and washed in PBS for 2 min 3 times. Slices were further incubated in 3\% freshly prepared $\mathrm{H}_{2} \mathrm{O}_{2}$ at room temperature for $10 \mathrm{~min}$ and washed in PBS for $2 \mathrm{~min} 3$ times. Fresh biotin solution was prepared, and $50 \mu \mathrm{L}$ labeling buffer was added to each sample, which were placed in the cartridge to keep the slices moist. Samples were then incubated at $37^{\circ} \mathrm{C}$ for $60 \mathrm{~min}$, washed in PBS for $2 \mathrm{~min}$, and $0.2 \mathrm{~mL}$ reaction-terminated liquid was added and further incubated at room temperature for $10 \mathrm{~min}$ and washed in PBS for 2 min 3 times.

Fifty microliters streptavidin-HRP working solution was added to each sample, placed in the cartridge to keep the slices moist, and incubated for $30 \mathrm{~min}$ at room temperature. Samples were washed in PBS for 2 min 3 times, and $0.3 \mathrm{~mL}$ DAB chromogenic solution was added, and incubated at room temperature for 30-60 min, which was deemed appropriate based on the incubation chromogenic results. Subsequently, samples were washed in PBS again 3 times, dehydrated, cleared by conventional graded alcohol, and mounted. Tissue sections were observed under a light microscope, and the brown cells were regarded as apoptotic cells. Eight different fields of view of each slice were randomly observed under high magnification and the average was calculated.

\section{Cytosolic and mitochondrial extracts of brain tissues}

Five rats from each group were killed and the brain tissues were taken after craniotomy. Approximately $100 \mathrm{mg}$ cerebral cortex was taken from the ischemic border zones. According to the literature (He et al., 2012), the specimens were placed in $0.6 \mathrm{~mL}$ homogenous media (0.25 M sucrose, $10 \mathrm{mM}$ Tris-HCl, $15 \mathrm{mM}$ EDTA, $0.2 \% \mathrm{BSA}, \mathrm{pH} 7.4)$ at $4{ }^{\circ} \mathrm{C}$, evenly homogenized 10 times with a glass homogenizer, treated by sonication on ice, and centrifuged at $12,000 \mathrm{~g}$ at $4^{\circ} \mathrm{C}$ for $10 \mathrm{~min}$. The supernatant was transferred to another centrifuge tube at $4^{\circ} \mathrm{C}$, and $0.3 \mathrm{~mL}$ medium was added to the homogenized sediment, which was repeatedly homogenized and then centrifuged at $12,000 \mathrm{~g}$ at $4^{\circ} \mathrm{C}$ for $10 \mathrm{~min}$. The two 
supernatants were merged, centrifuged at $12,000 \mathrm{~g}$ at $4^{\circ} \mathrm{C}$ for $10 \mathrm{~min}$, collected, and dispensed into five $0.5-\mathrm{mL}$ EP tubes and stored at $-80^{\circ} \mathrm{C}$ for cytosolic Cyt $\mathrm{C}$ testing. The precipitate was carefully collected (mitochondria). The mitochondrial deposits were suspended in $10 \mu \mathrm{L}$ preserved media (220 mM mannitol, $70 \mathrm{mM}$ sucrose, $10 \mathrm{mM}$ Tris, $1 \mathrm{mM}$ EDTA, pH 7.4) for MPTP opening testing.

\section{Detection of mitochondrial MPTP opening}

The UV-spectrophotometer was purchased from the Japan Tsushima Company. The mitochondrial swelling assay of the MPTP opening was carried out according to the kit instructions (Gene Haijie America Pharmaceutical Technology Co., Ltd.). The initial absorbance values in each group (A1) were measured at a 520 -nm wavelength, and $200 \mu \mathrm{M} \mathrm{CaCl}_{2}$ was added to induce MPTP opening; the absorbance values (A2) were recorded after $10 \mathrm{~min}$. The $\mathrm{A} 2 / \mathrm{A} 1$ ratio $(A)$ indicated the extent of MPTP opening.

\section{Western blot}

The protein electrophoresis system was provided by Bio-Rad (USA). One of the prepared EP tubes was used to measure the protein concentration by the bicinchoninic acid method. First, 5X sodium dodecyl sulfate (SDS) protein sample buffer was added at a 4:1 ratio and denatured in boiling water for $5 \mathrm{~min}$. The SDS-polyacrylamide gel electrophoresis (PAGE) was prepared, and the corresponding sample volume was calculated from $50 \mu \mathrm{g}$ denatured protein samples, which were loaded for electrophoresis for approximately $30 \mathrm{~min}$, first using an $80-\mathrm{V}$ constant voltage and then switching to a $120-\mathrm{V}$ constant voltage for approximately 60-90 min; SDS-PAGE was stopped when the bromophenol blue reached the bottom of the gel. The gel was transferred to a polyvinyldifluoride (PVDF) transmembrane at $4^{\circ} \mathrm{C}$ and $100 \mathrm{~V}$; the transmembrane time varied according to molecular weight. Tris-buffered saline with Tween 20 (TBST) with 5\% skim milk was used for sealing. The first antibody was antirabbit anti-mouse Cyt $\mathrm{C}$ mAb (Sigma, USA; 1:800 diluted), which was incubated overnight at $4^{\circ} \mathrm{C}$ at a dilution of 1:400 (Zhongshan Biotechnology, Beijing, China). The TBST-washed PVDF membrane was placed on a shaker 3 times for 10 min each time, and the secondary antibody, goat anti-rabbit (1:2000; Pierce, Rockford, IL, USA) was added and incubated for 3 $\mathrm{h}$ at room temperature. The membrane was washed in TBST three times, dyed with A and B mixed solution, transferred to an X-ray film tablet, and X-ray films were developed, fixed, and scanned. The images were saved in .tif format, and the gray values of the experimental results were analyzed using the Quantity-One software.

\section{Statistical analysis}

All data are reported as means \pm standard deviation, and the statistical analysis was performed using the SPSS 13.0 software. The comparison of data between multiple groups was performed using analysis of variance (ANOVA) when the data were normally distributed, and the least-square difference (LSD) method was selected to conduct pairwise comparisons for groups with homogenous variance; Games-Howell analysis was used when variance was heterogeneous. $\mathrm{P}<0.05$ indicated a statistically significant difference. 


\section{RESULTS}

\section{Quantitative analysis of experimental animals}

Sixteen models of 20 rats were successfully prepared; 1 rat scored 0 points and 2 rats scored 4 points in the initial neurological deficit test, and 1 rat died due to suffocation after postoperative gavage.

\section{Effect of atorvastatin intervention on MPTP opening of epencephalic tissues in MACO model rats}

The mitochondrial absorbance ratio $(A)$ did not differ significantly between the normal group and the sham-operation group $(\mathrm{P}>0.05)$. The $A$ value of the $\mathrm{I} / \mathrm{R}$ group was significantly reduced compared with the sham and normal groups $(\mathrm{P}<0.001)$, suggesting that the MPTP opening and increase in mitochondrial permeability were caused by ischemia-reperfusion. However, the $A$ value was significantly higher than that of the $\mathrm{I} / \mathrm{R}$ group after atorvastatin intervention $(\mathrm{P}<0.002)$, indicating that atorvastatin intervention could reduce the impact of ischemia-reperfusion on the MPTP and mitochondrial permeability (Figure 1).

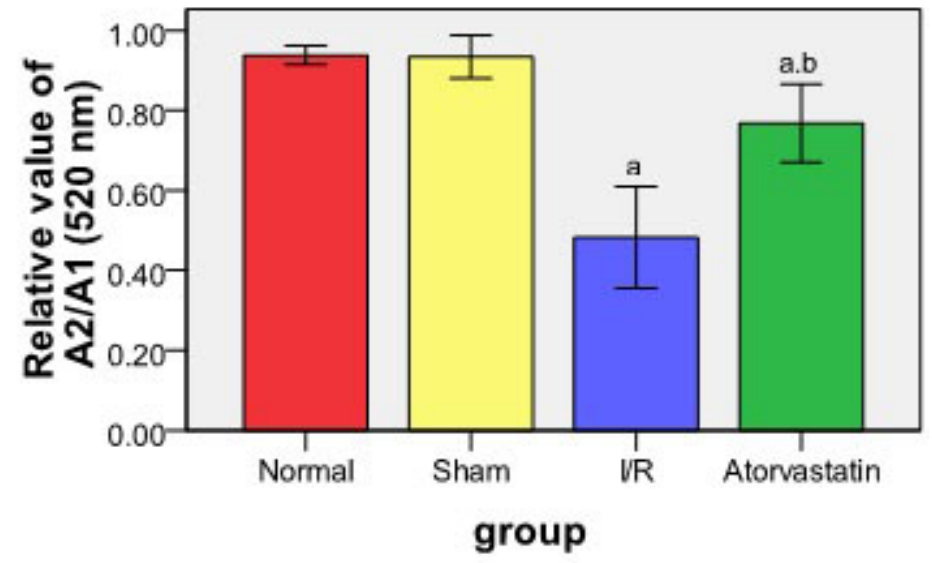

Figure 1. Effects of atorvastatin on MPTP opening in cerebral cortexes subjected to MACO. Data are reported as means $\pm \mathrm{SD}(\mathrm{N}=5)$. ${ }^{\mathrm{a}} \mathrm{P}<0.002$ vs normal or sham group; ${ }^{\mathrm{b}} \mathrm{P}<0.001$ vs $\mathrm{I} / \mathrm{R}$ group. I/R: ischemia/reperfusion; Sham: sham operation. A2/A1: absorbance ratio at $520 \mathrm{~nm}$.

\section{Effect of atorvastatin on Cyt C expression of epencephalic tissues in MACO model rats}

No expression of cytoplasmic Cyt $\mathrm{C}$ was found in the normal group and in the shamoperation group. The expression of cytoplasmic Cyt $\mathrm{C}$ was significantly increased in the $\mathrm{I} / \mathrm{R}$ group compared to the normal and the sham-operation groups $(\mathrm{P}<0.001)$. However, the expression of cytoplasmic Cyt $\mathrm{C}$ significantly decreased after atorvastatin intervention compared with the I/R group $(\mathrm{P}<0.001)$, indicating that atorvastatin intervention could reduce the release of Cyt C to the cytoplasm (Figure 2A and B). 
A

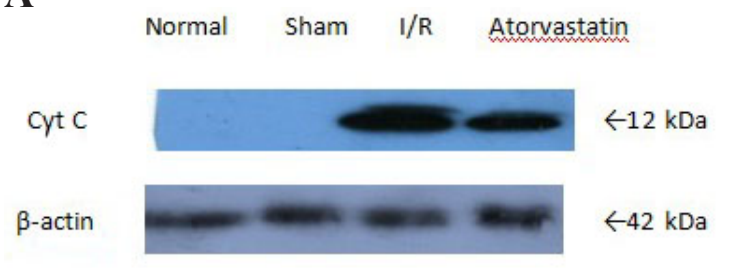

B

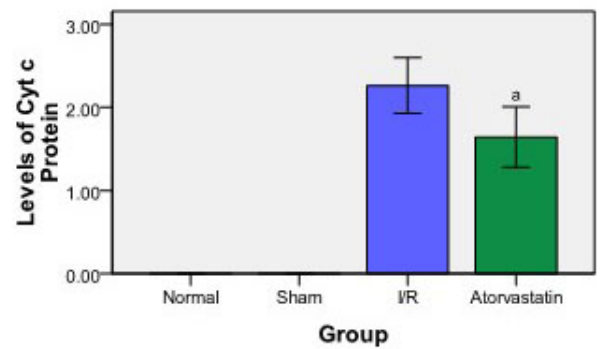

Figure 2. Effects of atorvastatin on the Cyt $\mathrm{C}$ protein in cerebral cortexes subjected to MACO. Data are reported as means $\pm \mathrm{SD}(\mathrm{N}=5) .{ }^{\text {a }}<0.001 v s \mathrm{I} / \mathrm{R}$ group. I/R: ischemia/reperfusion; Sham: sham operation. A. Electrophoresis strip of the Cyt $\mathrm{C}$ protein expressed in the brain cortex. Arrows indicate molecular mass. B. Levels of Cyt $\mathrm{C}$ protein in the cortex of different groups.

\section{Effect of atorvastatin intervention on apoptosis of epencephalic tissues in MACO model rats}

Few apoptotic cells were observed in the normal group and in the sham-operation group. The TUNEL-positive cells (nuclei tan or brown under high-magnification microscope) of the $\mathrm{I} / \mathrm{R}$ group were unevenly scattered throughout the entire ischemic penumbra and relatively concentrated in some regions, and the number of TUNEL-positive cells differed significantly from those in the normal group and the sham-operation group $(\mathrm{P}<0.001)$. The number of TUNEL-positive cells in the I/R group was significantly reduced compared with that of the atorvastatin intervention group $(\mathrm{P}<0.001)$, indicating that atorvastatin intervention could reduce apoptosis (Figure $3 \mathrm{~A}$ and $\mathrm{B}$ ).

\section{A}

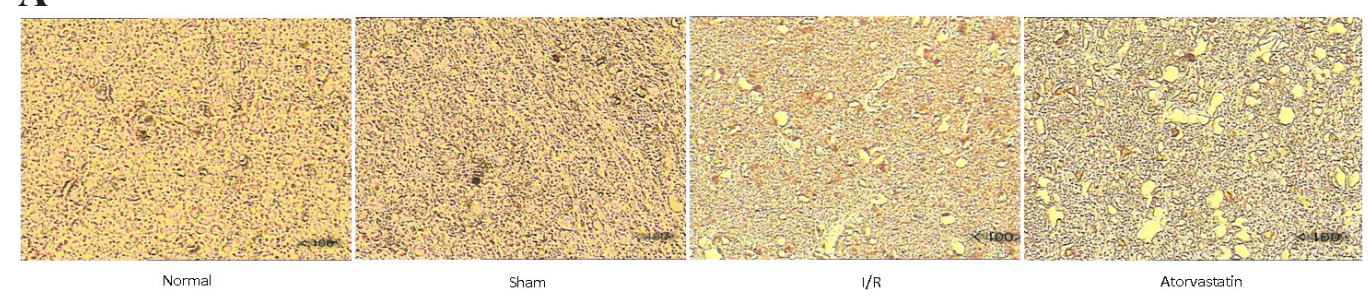

B

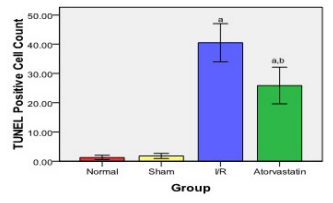

Figure 3. Effects of atorvastatin on neuronal apoptosis in cerebral cortexes subjected to MACO. Data are reported as means $\pm \mathrm{SD}$ (normal/sham group, $\mathrm{N}=2 ; \mathrm{I} / \mathrm{R}$ and atorvastatin group, $\mathrm{N}=5$ ). ${ }^{\mathrm{P}}<0.001$ vs normal or sham group; ${ }^{\mathrm{P}}<0.001$ vs I/R group. I/R: ischemia/reperfusion; Sham: sham operation; TUNEL: terminal deoxynucleotidyltransferase (TdT)-mediated dUTP nick-end labeling. A. Photomicrographs of TUNEL staining in the brain cortex (400X). Arrows indicate apoptotic neurons. B. Numbers of TUNEL-positive neurons were counted in 8 visual fields of each section (400X). 


\section{Effect of atorvastatin intervention on nerve cell morphology of epencephalic tissues in MACO model rats}

The HE staining results showed that the brain tissue structures of the normal group and the sham-operation group were normal, and the nerve cells were tightly packed with abundant cytoplasm and clear nucleoli. The nerve cells of the I/R group appeared sparse and irregular, showing cell shrinkage and pyknotic-stained nuclei, and some nuclei even disappeared. These changes were abated in the atorvastatin intervention group (Figure 4).

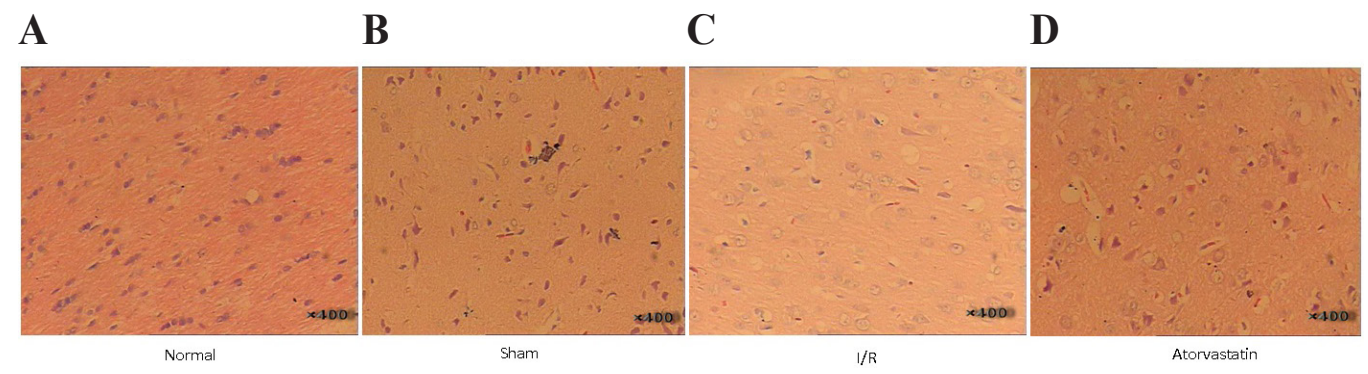

Figure 4. Effects of atorvastatin on the cellular morphology of cerebral cortexes subjected to MACO. I/R: ischemia/ reperfusion; Sham: sham operation. A.-D. Photomicrographs of HE-staining in the brain cortex (400X).

\section{DISCUSSION}

Cerebral ischemia-reperfusion injury is a complex pathophysiological process. Previous studies have found that the ischemia-reperfusion injury of the epencephalic tissues was related to rapid cascade reactions, such as oxygen free radicals, excitatory amino acids, release of inflammatory mediators, calcium overload, and apoptosis gene activation. Therefore, it has been a challenge to find protective treatment for brain tissues from ischemic injury in clinical settings.

Atorvastatin is the second generation of HMG-CoA reductase inhibitors, and is mainly metabolized through the liver or outward liver. In addition to the traditional function of lowering low-density lipoprotein cholesterol levels, atorvastatin also has cholesterol-independent pleiotropic biological effects, and its neuroprotection effects have received increasing attention, in particular. Hong et al. (2006) pretreated SD rats with $10 \mathrm{mg} / \mathrm{kg}$ atorvastatin three times before cerebral ischemia-reperfusion, and found that atorvastatin could protect the ischemic penumbra of nerve cells by inhibiting NADPH oxidase-derived superoxide, thereby reducing the infarct volume. Furthermore, Cui et al. (2010) administered atorvastatin to rats with acute phase cerebral ischemia and found that the blood brain barrier permeability of rats significantly increased, brain edema decreased, infarct volume decreased. In addition, the protective mechanism was found to be associated with the reduction in the expressions of 12/15-LOX, p38MAPK, and cPLA2 by immunohistochemistry, RT-PCR, and Western blot analysis, which showed dose-dependent effects. The protective effect of atorvastatin on ischemic brain tissues was also associated with p120 increase, expression of $\alpha \mathrm{N}$-interlink synaptic adhesion protein, and improvement in synaptic plasticity (Céspedes-Rubio et al., 2010). These studies evaluated the short-term effects of atorvastatin, whereas Yrjanheikki et al. (2005) observed permanent middle cerebral artery occlusion of a rat group and found that atorvastatin still had a neuropro- 
tective effect after 21 days. These studies demonstrated that atorvastatin had a definite protective effect on ischemic brain tissues at different levels. However, previous studies have mainly concentrated on the expression of cytoplasm proteins, and few have investigated the influence of atorvastatin on cerebral ischemia-reperfusion injury in rats at the subcellular organelle (i.e., mitochondria, endoplasmic reticulum, Golgi apparatus) level.

In this study, after cerebral ischemia for $2 \mathrm{~h}$ and reperfusion for $72 \mathrm{~h}$ and HE staining, the morphological changes of nerve cells in the $\mathrm{I} / \mathrm{R}$ group were reduced compared to those in the atorvastatin intervention group, which confirmed the neuroprotective effect of atorvastatin in ischemia-reperfusion brain tissues shown in previous studies (Yrjanheikki et al., 2005; Hong et al., 2006; Céspedes-Rubio et al., 2010; Cui et al., 2010). We found that the apoptosis rate of the atorvastatin intervention group was significantly reduced compared with that of the I/R group by TUNEL staining, suggesting that the neuroprotective effect of atorvastatin was related to cell apoptosis inhibition.

Previous studies have shown that the effect of apoptosis is influenced by various factors, including the membrane receptor pathway, the mitochondrial pathway, protease B particles, and the endoplasmic reticulum pathway (Łabedzka et al., 2006). In the cellular environment, the combination of the endogenous pathway and the extrinsic pathway could consequently cause a series of pathophysiological changes. The intrinsic pathway is mainly triggered by mitochondria. Mitochondria not only generate the necessary ATP to maintain ion pump function and to ensure stability of the internal environment, but also play important roles in physiological cellular signal transduction and pathophysiological events (Cai et al., 1998; Ichas and Mazat, 1998). After cerebral ischemia-reperfusion, due to the release of inflammatory mediators and oxygen-free radicals, the toxic effects of excitatory amino acids, calcium overload, apoptotic gene activation, and a series of other rapid cascades of the intracellular environment, the nerve becomes disordered, thus promoting the apoptosis pathway of mitochondria.

MPTP is a transmembrane channel protein, which is essentially located between the inner and outer mitochondrial membrane. The key point of the information exchange within and outside of the mitochondria mainly involves non-selective complexes including the voltage-dependent anion channel, adenylate transposons, constitutive cyclophilin D, and other substances, which are sometimes referred to as mitochondrial irritant-receptors (Javadov et al., 2011). The periodic opening plays an important role in maintaining the steady state and electrochemical balance of mitochondria. MPTP in the inner mitochondrial membrane protein is activated upon oxidative stress or calcium overload, resulting in the uncoupling of oxidative phosphorylation, osmotic swelling, and rupture of the outer mitochondrial membrane, thereby releasing large amounts of Cyt C (Halestrap et al., 2000). Cyt C is a water-soluble protein located in the outer mitochondrial intima among the respiratory chain complex III-IV, and plays an important role in mitochondrial energy metabolism as an electron transfer carrier of the respiratory chain (Lenaz and Genova, 2010). Cyt C binds to apoptosis activating factor-1 (Apaf-1) after being released into the cytoplasm and forms a composite body (molecular weight, 700-1400 x 103), which then activates Caspase-9, forming a Cyt C/Apaf-1/Caspase-9 complex, i.e., the apoptotic body complex. With the reduction or dysfunction of $\mathrm{Cyt} \mathrm{C}$, the mitochondrial respiratory chain becomes disrupted, and ATP synthesis is decreased, eventually leading to cell death (Li et al., 1997). According to the degree of MPTP opening and the release rate of Cyt $\mathrm{C}$, the cell death mode was divided into rapid necrosis (-ATP) or delayed apoptosis (+ATP) (Orrenius, 2004; Saikumar et al., 2007). Therefore, in the process of brain tissue ischemia-reperfusion injury, the cell death mode can be changed by inhibiting the MPTP 
opening, reducing the release of Cyt $\mathrm{C}$, and inhibiting the formation of the apoptotic body complex, which may ultimately save the cells close to death. In this study, atorvastatin was found to inhibit $\mathrm{CaCl}_{2}$-induced MPTP swelling by treating the cerebral tissues after ischemiareperfusion and to increase the experimental absorbance values of the I/R group. In addition, the release of cytoplasmic Cyt $\mathrm{C}$ also decreased compared to that of the $\mathrm{I} / \mathrm{R}$ group, suggesting that atorvastatin had effects on specific sites of the MPTP through the blood brain barrier, changed the permeability of the mitochondrial transmembrane channels, inhibited mitochondrial swelling, and thereby protected mitochondrial structure and function and reduced the pathway of cell apoptosis in mitochondria.

Together, these results suggest that atorvastatin could inhibit neuronal apoptosis and reduce the ischemia-reperfusion injury of brain tissues, and that the mechanism may be associated with the blocking of the MPTP opening and the consequent reduction of Cyt $\mathrm{C}$ release. Due to the complex apoptosis pathway involving several interweaving and intercommunicating factors, the investigation of changes in other subcellular organelles and apoptotic factors may contribute to further understanding of the acting mechanism of atorvastatin.

\section{ACKNOWLEDGMENTS}

Research supported by the Hunan Province Medical Research Foundation (\#B2010-090).

\section{REFERENCES}

Bösel J, Gandor F, Harms C, Synowitz M, et al. (2005). Neuroprotective effects of atorvastatin against glutamate-induced excitotoxicity in primary cortical neurons. J. Neurochem. 92: 1386-1398.

Cai J, Yang J and Jones DP (1998). Mitochondrial control of apoptosis: the role of cytochrome c. Biochim. Biophys. Acta 1366: 139-149.

Céspedes-Rubio A, Jurado FW and Cardona-Gómez GP (2010). p120 catenin/ $\alpha \mathrm{N}$-catenin are molecular targets in the neuroprotection and neuronal plasticity mediated by atorvastatin after focal cerebral ischemia. J. Neurosci. Res. 88: 3621-3634.

Clark WM, Lessov NS, Dixon MP and Eckenstein F (1997). Monofilament intraluminal middle cerebral artery occlusion in the mouse. Neurol. Res. 19: 641-648.

Cui L, Zhang X, Yang R, Wang L, et al. (2010). Neuroprotection of early and short-time applying atorvastatin in the acute phase of cerebral ischemia: down-regulated 12/15-LOX, p38MAPK and cPLA2 expression, ameliorated BBB permeability. Brain Res. 1325: 164-173.

Halestrap AP, Doran E, Gillespie JP and O'Toole A (2000). Mitochondria and cell death. Biochem. Soc. Trans. 28: 170-177.

He XL, Bi MG and Du GH (2012). Brain mitochondria proteome and energy metabolism in rats after chronic cerebral hypoperfusion. Chin. Pharmacol. Bull. 28: 1200-1205.

Hong H, Zeng JS, Kreulen DL, Kaufman DI, et al. (2006). Atorvastatin protects against cerebral infarction via inhibition of NADPH oxidase-derived superoxide in ischemic stroke. Am. J. Physiol. Heart Circ. Physiol. 291: H2210-H2215.

Ichas F and Mazat JP (1998). From calcium signaling to cell death: two conformations for the mitochondrial permeability transition pore. Switching from low- to high-conductance state. Biochim. Biophys. Acta 1366: 33-50.

Javadov S, Hunter JC, Barreto-Torres G and Parodi-Rullan R (2011). Targeting the mitochondrial permeability transition: cardiac ischemia-reperfusion versus carcinogenesis. Cell. Physiol. Biochem. 27: 179-190.

Łabedzka K, Grzanka A and Izdebska M (2006). Mitochondria and cell death. Postepy. Hig Med. Dosw. 60: 439-446.

Lenaz G and Genova ML (2010). Structure and organization of mitochondrial respiratory complexes: a new understanding of an old subject. Antioxid. Redox. Signal. 12: 961-1008.

Li P, Nijhawan D, Budihardjo I, Srinivasula SM, et al. (1997). Cytochrome c and dATP-dependent formation of Apaf-1/ caspase-9 complex initiates an apoptotic protease cascade. Cell 91: 479-489.

Longa EZ, Weinstein PR, Carlson S and Cummins R (1989). Reversible middle cerebral artery occulusion without craniectomy in rats. Stroke 20: 84-91.

Nahrendorf M, Sosnovik D, Chen JW, Panizzi P, et al. (2008). Activatable magnetic resonance imaging agent reports 
myeloperoxidase activity in healing infarcts and noninvasively detects the antiinflammatory effects of atorvastatin on ischemia-reperfusion injury. Circulation 117: 1153-1160.

Orrenius S (2004). Mitochondrial regulation of apoptotic cell death. Toxicol. Lett. 149: 19-23.

Pignatelli P, Carnevale R, Pastori D, Cangemi R, et al. (2012). Immediate antioxidant and antiplatelet effect of atorvastatin via inhibition of Nox2. Circulation 126: 92-103.

Saikumar P, Mikhailova M and Pandeswara SL (2007). Regulation of caspase-9 activity by differential binding to the apoptosome complex. Front Biosci. 12: 3343-3354.

Ye R, Yang Q, Kong X, Li N, et al. (2012). Sevoflurane preconditioning improves mitochondrial function and long-term neurologic sequelae after transient cerebral ischemia: role of mitochondrial permeability transition. Crit. Care Med. 40: 2685-2693.

Yrjanheikki J, Koistinaho J, Kettunen M, Kauppinen RA, et al. (2005). Long-term protective effect of atorvastatin in permanent focal cerebral ischemia. Brain Res. 1052: 174-179.

Zhang Y, Huang S, Wang B, Sun B, et al. (2012). Atorvastatin and whisker stimulation synergistically enhance angiogenesis in the barrel cortex of rats following focal ischemia. Neurosci. Lett. 525: 135-139.

Zhao Q, Wang S, Li Y, Wang P, et al. (2013). The role of the mitochondrial calcium uniporter in cerebral ischemia/ reperfusion injury in rats involves regulation of mitochondrial energy metabolism. Mol. Med. Rep. 7: 1073-1080. 